\title{
Long range local effective potential for a three-particle system
}

\author{
A. Delfino \\ Departamento de Física, Universidade Federal Fluminense, 24210 Niterói, Rio de Janeiro, Brazil \\ T. Frederico \\ Instituto de Estudos Avançados, Centro Técnico Aeroespacial, 12200 São José dos Campos, São Paulo, Brazil \\ Lauro Tomio \\ Instituto de Física Teórica, Universidade Estadual Paulista, 01405 São Paulo, São Paulo, Brazil
}

(Received 2 March 1988)

\begin{abstract}
We derive the long- range behavior of an effective interaction for a system with three identical particles. We start our derivation from the zero range theory in configuration space and we show that the asymptotic effective potential, in the relative distance of the subsystem to the third particle, goes as $-\exp (-\lambda r) /\left(r^{3 / 2}\right)$.
\end{abstract}

It has been shown recently that the observed correlation connecting binding energies and scattering lengths in three-nucleon systems, known as the Phillips plot, ${ }^{1}$ can essentially be explained in a nucleon-deuteron $(n-d)$ optical potential model. ${ }^{2}$ Correlations between the doublet $n-d$ scattering length $a_{n d}$ and the energy of the excited virtual state of the triton were also pointed out by Girard and Fuda. ${ }^{3}$ They have also observed that the triton binding energy and the asymptotic normalization parameter (ANP) are linearly correlated. Several other works have discussed also the strong correlation among low-energy three-nucleon observables ${ }^{4}$ and more recently correlations between $n$ - $d$ observables above the breakup threshold have been discussed ${ }^{5}$ as well. In Ref. 2 , it was pointed out that it is possible to introduce a simple nucleondeuteron optical potential if one does not limit oneself to the usual class of short-range potentials. The main problem is associated with the very small binding energy of the deuteron on the nuclear scale and correspondingly with the extension of its wave function in coordinate space. Because of the large size of the deuteron, the onenucleon-exchange part of the nucleon-deuteron potential has an attractive long-range tail. Two nucleons in the trinucleon system experience an effective interaction well outside the range of the nucleon-nucleon interaction. The first suggestion of the effective long-range potential was given by Efimov, ${ }^{6}$ who showed that an effective three-body interaction of $\sim 1 / \rho^{2}$, where $\rho$ is the hyperradius, arises for a three-boson system when the two-body binding energy $\left(E_{2}\right)$ goes to zero. This potential gives rise to the infinite number of bound states of the three boson system, known as the Efimov effect. Later Fonseca, Redish, and Shanley (FRS) (Ref. 7) showed, in the BornOppenheimer approximation, that the effective potential between two heavy particles, interacting through the exchange of a light particle, has a long-range $1 / R^{2}$ tail as the light-heavy binding energy reduces to zero. Here $R$ is the relative distance between the two heavy particles. This $1 / R^{2}$ interaction is essential in order to yield the Efimov effect ${ }^{6}$ in this case. Although these works do not obtain a potential for the $n$ - $d$ system, the suggested effective nucleon-deuteron potential used in Ref. 2 was based on the approach of Refs. 6 and 7.

Our task in this work is to derive the tail of the effective two-body interaction for a system of three identical particles for $E_{2}$ nonzero, a goal that was only suggested in previous works. We then derive the asymptotic behavior of the local effective two-body potential for a system consisting of three identical spinless particles as well as that for the case of a spin quartet three-nucleon system.

We start our derivation from an ansatz based on the zero-range theory ${ }^{8}$ for the three-particle bound-state wave function. The ansatz for the wave function implies the existence of a corresponding three-particle binding energy; when this assumption is not correct, as in the case of the spin quartet three-nucleon system, we hope that the resulting effective potential will reflect such a situation. The projection of the three-particle bound-state wave function onto the two-body bound state gives rise to an effective two-body wave function for the three-particle system. Our basic assumption is that a local effective potential for the relative motion can be derived from the Schrödinger equation when the wave function has zero orbital angular momentum in the relative coordinate. This implies that our effective local potential is expected to satisfy the following equation:

$$
V(r)=\frac{3}{4}\left[\frac{d^{2}}{d r^{2}} \chi(r)-\mu^{2} \chi(r)\right] / \chi(r),
$$

where $\chi(r) / r$ is the effective two-body radial wave function for the three-particle system, $r$ is the distance from the center of mass of a pair to the third particle, and $\mu^{2}=\frac{4}{3}\left(E_{3}-E_{2}\right)$ with $E_{3}$ the three-particle binding energy. The units we are using are such that $\hbar$ and the masses of the particles are equal to one.

The three-particle bound-state wave function is given by an approximate solution of the Schrödinger equation 
in the zero-range limit. ${ }^{8}$ In this case, the Faddeev component of the wave function is given by

$$
\begin{aligned}
\psi(\mathbf{r}, \mathbf{R})=\int & d^{3} q \exp (\mathbf{i q} \cdot \mathbf{r}) \varphi(\mathbf{q}) \\
& \times \int d^{3} p \frac{\exp (i \mathbf{p} \cdot \mathbf{R})}{\left(E_{3}+\frac{3}{4} q^{2}+p^{2}\right)},
\end{aligned}
$$

where $R=|\mathbf{R}|$ is the relative distance between the particles of the interacting pair. We assume $\varphi(\mathbf{q})=\left(\mu^{2}+q^{2}\right)^{-1}$ in order to obtain the correct asymptotic behavior of $\psi$ for $r \rightarrow \infty$. These are clearly the minimal assumptions that one must make when defining the three-body wave function in the zero-range limit. The expression obtained for $\psi$ has already been used to explain several triton $D$-state properties (see Ref. 9). Now, to arrive at the effective two-body radial wave function in its reduced form, we project the total three-body wave function onto the radial bound-state wave function of the interacting pair. The state of the interacting pair has a Yukawa form as it is imposed by the free Schrödinger equation with the appropriate boundary conditions. This gives us

$$
\begin{aligned}
\chi(r)= & \int_{-\infty}^{\infty} \frac{d q q \sin q r}{\left(\mu^{2}+q^{2}\right)\left[\left(E_{3}+3 q^{2} / 4\right)^{1 / 2}+\sqrt{\left.E_{2}\right]}\right.} \\
& +\gamma \frac{4}{3 \pi} \int_{-\infty}^{\infty} \frac{d q \sin q r}{\left(\mu^{2}+q^{2}\right)} \int_{-\infty}^{\infty} \frac{d k k}{\left(\mu^{2}+k^{2}\right)} \ln \left[\frac{E_{3}+3 q^{2} / 4+(k-q / 2)^{2}}{E_{2}+(k-q / 2)^{2}}\right),
\end{aligned}
$$

where we drop an unnecessary overall factor.

The first integral of Eq. (3), the direct term $\left(\chi_{d}\right)$, comes from the projection, onto the two-body bound-state wave function, of the Faddeev component, Eq. (2), that has motion in the same coordinate $\mathbf{R}$ that appears in the two-body wave function. The second term of Eq. (3) we call the exchange term $\left(\chi_{e}\right)$ as it comes from the projection (onto the same two-body wave function) of the other Faddeev components having motion in coordinates of the interacting pair other than that of the two-body wave function. The $\gamma$ factor that appears multiplying this second term is given by the symmetry of the system; $\gamma=2$ for the three-boson case and $\gamma=-1$ for the spin-quartet three-nucleon system. As pointed out by Efimov ${ }^{6}$ the strength of the long-range potential must depend on the symmetry of the wave function, and one should expect to have less attraction in the case of $\gamma=-1$.

For $E_{2}$ not equal to zero, it is not difficult to derive the asymptotic dominance of the direct term:

$$
\chi(r) \stackrel{r \rightarrow \infty}{\rightarrow} \pi \exp (-\mu r) /\left(2 \sqrt{E_{2}}\right)
$$

To obtain this behavior, it is enough to perform a contour integration in the upper complex plane. The exponential decreasing tail appears as the residue of the integral for $q=i \mu$, when $E_{2} \neq 0$. This residue is the only term that asymptotically survives in Eq. (3). It is greater than the other integral that comes from the direct term. The exchange term does not have such an exponential tail, as the pole is canceled by the logarithmic functions.

When $E_{2}$ is zero this argument is no longer true, as different terms from the direct and exchange part alter such behavior. This gives origin to a long-range potential (see Appendix).

In Eq. (4) we have determined the exponential decreasing behavior of the denominator of Eq. (1) in the asymptotic limit. The next step is to study the numerator of Eq. (1) in the same limit. To obtain the contribution of the direct term of Eq. (3) to the numerator of Eq. (1), we perform an analytical extension of the path of the integration to the upper complex plane. As a consequence of the square-root cut, we have an integral whose path is part of the imaginary axis. Then it is easy to perform the limit $r \rightarrow \infty$ :

$$
\begin{aligned}
\frac{d^{2}}{d r^{2}} \chi_{d}(r)-\mu^{2} \chi_{d}(r) & =-\frac{4}{\sqrt{3}} \int_{\sqrt{4 E_{3} / 3}}^{\infty} y d y \exp (-y r) \frac{\left(y^{2}-4 E_{3} / 3\right)^{1 / 2}}{y^{2}-\mu^{2}} \\
& \rightarrow \infty \\
& \rightarrow-\frac{2 \sqrt{\pi}}{3 E_{2}}\left(\frac{\sqrt{3 E_{3}}}{r}\right)^{3 / 2} \exp \left(-2 r \sqrt{E_{3} / 3}\right)
\end{aligned}
$$

In order to obtain the asymptotic contribution of the exchange term to the effective potential, we first introduce $\chi_{e}(r)$ in the numerator of Eq. (1) and then integrate by parts in the $q$ variable. This yields:

$$
\begin{aligned}
\frac{d^{2}}{d r^{2}} \chi_{e}(r)-\mu^{2} \chi_{e}(r)= & -\frac{8 \pi}{3 r} \exp \left[-2 r\left(\mu+\sqrt{\left.E_{2}\right)}\right]\right. \\
& +\frac{8}{3 r} \int_{-\infty}^{\infty} d x \frac{x \sin (x / 2)}{x^{2}+(\mu r)^{2}} \exp \left[-\left(E_{3} r^{2}+3 x^{2} / 4\right)^{1 / 2}\right] .
\end{aligned}
$$


Here we find that, in the limit $r \rightarrow \infty$, it is reasonable to drop the term $x^{2}$ in the pole term of Eq. (6). The resulting expression is an integral form of the modified Bessel function of imaginary argument. By using the asymptotic expression for the modified Bessel function, we obtain our result for the asymptotic limit of the exchanged part, which has the same analytical form as the direct term. The first term in Eq. (6) decrease faster than the second one as $r$ goes to infinity, so only the former is needed in this limit. Therefore, we can already sum this last result with Eq. (5) to obtain the numerator of Eq. (1). By introducing Eq. (4) in Eq. (1), we obtain the final expression for the long-range part of the effective potential:

$$
\begin{array}{r}
\left.V(r) \stackrel{r \rightarrow \infty}{\rightarrow}-\frac{8}{3}\left(\frac{2}{\pi}\right)^{1 / 2} \frac{E_{3}^{3 / 4}}{E_{2}^{1 / 2}} \mid 1+\gamma \frac{2 E_{2}}{\sqrt{3} \mu^{2}}\right) \\
\times \frac{\exp \left[-\left(\sqrt{4 E_{3} / 3}-\mu\right) r\right]}{r^{3 / 2}} .
\end{array}
$$

This equation is the main result of the present work. It is clear that there is an increase of the intensity of the effective interaction for the three-boson case $\gamma=2$ in relation to the spin-quartet $n-d$ system $(\gamma=-1)$, in agreement with the findings of Efimov. ${ }^{6}$ We note that the argument of the exponential in Eq. (7) reflects the size of the two-body bound-state wave function. Our result for the asymptotic dependence of the potential cannot be extended to the limit $E_{2}=0$ as Eq. (7) is no longer valid in this limit (see Appendix).

Our derivation was based on minimal assumptions on the wave function that must be valid for any short-range two-particle interaction. This supports the model independence of the long-range part of the effective potential. This feature has already been discussed in Refs. 2 and 10 in connection with the correlations between three-nucleon low-energy observables. We observe that, although the form of $V(r)$ used in Ref. 2 is different from our result, both have in common the same important qualitative feature of the potential, namely the range associated with the size of the deuteron. In addition, we have obtained explicitly the energy dependence of the parameters of the effective two-body potential. This also is in agreement with the suggestion by Noyes. ${ }^{11}$

In conclusion, we obtained the long-range part of the effective two-body potential for a three-boson system in the relative $S$ state in configuration space for $E_{2}$ nonzero. We give a close expression for this potential, with the range and intensity as a function of the three- and twobody bound-state energies and the symmetry of the total wave function. It will be interesting to test the above findings in a numerical study of the three-nucleon system. This will be investigated in a future work.

\section{ACKNOWLEDGMENTS}

Discussions with S. K. Adhikari, C. E. I. Carneiro, B. V. Carlson, I. D. Goldman, and C. H. Lewenkopf are appreciated. This work was supported by the Conselho Nacional de Desenvolvimento Científico e Tecnológico.

\section{APPENDIX}

The derivation of the asymptotic dependence of the effective potential, for the case $E_{2}=0$, is accompanied by a difficulty associated with the resolution of the integral which defines the exchange term. For simplicity, we study only the direct term of Eq. (3), which is just an integral representation of the modified Bessel function $K_{0}(\mu r)$ :

$$
\chi_{d}(r)=\frac{4 r}{\sqrt{3}} K_{0}(\mu r) .
$$

The asymptotic form for the potential is obtained by using Eq. (A1) in Eq. (1) together with the asymptotic behavior of $K_{v}(\mu r)$ :

$$
V(r)=-\frac{3 \mu}{4 r} \frac{K_{1}(\mu r)}{K_{0}(\mu r)} \stackrel{r \rightarrow \infty}{\rightarrow}-\left(\frac{3 \mu}{4 r}+\frac{3}{8 r^{2}}\right)
$$

In Eq. (A2), the tail $\mu / r$ has nothing to do with the usual Coulomb interaction. $V^{\prime}=-3 \mu / 4 r$ alone does not support any bound state as the three-body energyparameter $\mu$ destroys completely the acceptable physical behavior of the wave function. In this context $V^{\prime}$ has to be taken just as a perturbation on $V_{0}=-3 / 8 r^{2}$, the only relevant term in Eq. (A2) in order to generate the infinite number of bound states prescribed by Ref. 6 . Comparing our $V(r)$ with that obtained by Efimov ${ }^{6}$ in a different approach, we have an additional perturbing term $V^{\prime}$ which does not modify his conclusions about the number of three-particle bound states for $E_{2}=0$. Another comparison can be made with the effective two-body potential derived by FRS. ${ }^{7}$ To obtain consistency between our Eq. (A2) and Eq. (37) of FRS, we only have to observe that one should have $E_{2} \sim E_{3}$ in order for the adiabatical approximation to be valid.
${ }^{1}$ A. C. Phillips, Phys. Rev. 142, 984 (1985); Nucl. Phys. A107, 209 (1968); Rep. Prog. Phys. 40, 905 (1977).

${ }^{2}$ L. Tomio, A. Delfino, and S. K. Adhikari, Phys. Rev. C 35, 441 (1987); S. K. Adhikari, A. Delfino, and L. Tomio, in Proceedings of the International Workshop on Few-Body Approaches to Nuclear Reactions in Tandem and Cyclotron Energy Regions, edited by S. Oryu and T. Sawada (World-Scientific, Singa- pore, 1987), p. 52.

${ }^{3}$ B. A. Girard and M. G. Fuda, Phys. Rev. C 19, 579 (1979); 19, 583 (1979).

${ }^{4}$ S. K. Adhikari, Phys. Rev. C 30, 31 (1984) and Refs. therein; D. D. Brayshaw, Phys. Rev. Lett. 32, 382 (1984).

${ }^{5}$ T. Frederico and I. D. Goldman, Phys. Rev. C 37, 1661 (1987). ${ }^{6}$ V. Efimov, Phys. Lett. 33B, 563 (1970). 
${ }^{7}$ A. C. Fonseca, E. F. Redish, and P. E. Shanley, Nucl. Phys. A320, 273 (1979); see also Yu. N. Ovchinnikov and I. M. Sigal, Ann. Phys. 123, 274 (1979).

${ }^{8}$ G. V. Skorniakov and K. A. Ter-Martirosian, Zh. Eksp. Teor. Fiz. 31, 775 (1956). [Sov. Phys. -JETP 4, 648 (1957)]; H. P.
Noyes, Phys. Rev. C 26, 1858 (1982).

${ }^{9}$ T. Frederico, S. K. Adhikari, and M. S. Hussein, Phys. Rev. C 37, 364 (1988).

${ }^{10}$ V. Efimov, Nucl. Phys. A362, 45 (1981).

${ }^{11}$ H. P. Noyes, SLAC Report PUB-4321, 1987. 